\title{
Analysis and Design of a Current-fed Two Inductor Bi-directional DC/DC Converter using Resonance for a Wide Voltage Range
}

\author{
Yong-Su Noh*, Bum-Jun Kim*, Sung-Chon Choi*, Do-Yun Kim* and Chung-Yuen Won ${ }^{\dagger}$
}

\begin{abstract}
In this paper, a current-fed two-inductor bi-directional DC/DC converter using resonance (CF-TIBCR) and its design method are proposed. The CF-TIBCR has characteristics of low current ripple and a high current rating because of two separated inductors. Also, it achieves zero voltage switching for all switches and zero current switching for switches of a low voltage stage by using the resonant tank. Besides, a voltage spike problem in conventional current-fed converters is solved without the need for an additional snubber or clamping circuits. As a result, the CF-TIBCR features high step-up and high efficiency. Since the proposed converter has difficulty achieving the softswitching condition when the converter requires the low voltage transfer ratio, a method that varies the number of resonant cycles is adopted to extend the output voltage range with satisfying the softswitching condition. The principles of the operation characteristics are presented with a theoretical analysis, and the proposed converter is verified through results of an experiment using a laboratory prototype.
\end{abstract}

Keywords: Current-fed DC/DC converter, Zero voltage switching (ZVS), Zero current switching (ZCS), Resonance, Wide voltage range

\section{Introduction}

Recently, battery, photovoltaics (PV) and fuel-cells (FC) have received much attention as sources of energy that can solve the problems of environmental pollution and energy exhaustion. These types of energy sources are characterized by low voltage and high current output. Therefore, DC/DC converters for these power sources are needed to achieve a high step-up characteristic, low current ripple and galvanic isolation [1-3]. The current-fed twoinductor boost converter (CF-TIBC), which is derived from the duality principle in 1993 by using a transformer-coupled half-bridge converter, is suitable for these applications $[4,5]$. The CF-TIBC has inherent high reliability and a step-up characteristic, and it can obtain a high input current rating and low input current ripple because the input current flows into the two separated inductors [4-16].

However, the CF-TIBC has several drawbacks when using the battery applications. Firstly, the CF-TIBC is not capable of charging a battery due to a unidirectional converter. Battery applications, such as an energy storage system (ESS) and electric vehicles (EVs) can require a bidirectional power flow to charge or to discharge a battery. Some research has contributed to the operation of the CF-TIBC using two opposite directions; this has involved replacing diodes with active switches [13-20].

$\dagger$ Corresponding Author: School of Electronic and Electrical Engineering, Sungkyunkwan University, Suwon, Korea. (woncy550@gmail.com)

* School of Electronic and Electrical Engineering, Sungkyunkwan University, Suwon, Korea. (\{nys66, bushat, chon8787, kdy1279\}@ skku.edu)

Received: July 22, 2015; Accepted: June 27, 2016
The other problem of the conventional CF-TIBC leads to a high voltage spike when the switches are turned off. The voltage spike occurs due to the reverse recovery current of secondary diodes and unavoidable transformer leakage inductance; this is the reason why the conventional CF-TIBC is limited to a switching frequency and power rating. To address these problems, additional snubbers or active clamp circuits have been researched [6-8, 21]. However, these methods lead to rising production cost and complications of the circuit and deteriorating reliability of the system.

In this paper, a current-fed two-inductor bi-directional $\mathrm{DC} / \mathrm{DC}$ converter using resonance (CF-TIBCR) is proposed as shown in Fig. 1. The proposed converter is mainly composed of two stages, which are a low voltage (LV) and a high voltage (HV) stage separated by the transformer. The diodes of the HV stage are replaced with the MOSFET switches. Thus, it is possible to conduct a bi-directional operation. Using the resonant components of the LV stage, the switches of the LV stage are satisfied with a zero voltage switching (ZVS) condition and a zero current switching (ZCS) when the switches are turned-on and

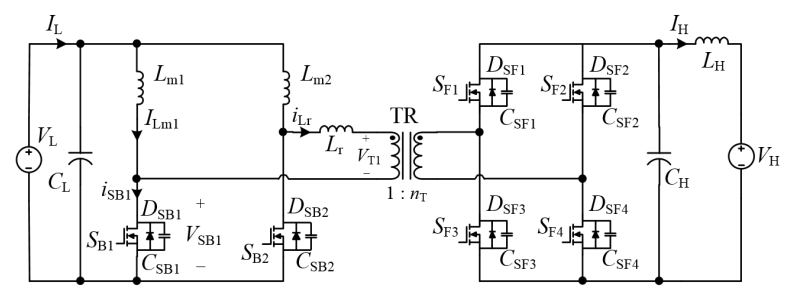

Fig. 1. Schematic of the proposed CF-TIBCR 
turned-off, respectively [9-16]. The switches of the HV stage also achieve ZVS. Therefore, the voltage spike problem is eliminated and system efficiency can be increased. The output voltage of the proposed converter is determined by the switching frequency, the resonant frequency, and the number of the resonant cycles. When the CF-TIBCR requires a low voltage transfer ratio, it is hard to satisfy the soft-switching condition. Therefore, a method of varying the number of the resonant cycles is adopted to increase the output voltage range and this makes soft switching possible within the entire operating range.

An introduction of the converter and a description of the operation modes and principles are presented in Section 2. Section 3 explains characteristics of the CFTIBCR according to the number of the resonant cycles, and the design method of the resonant component. Then, experimental results for a wide voltage range and load variation using a $300 \mathrm{~W}$ laboratory prototype are demonstrated in Section 4 in order to verify the proposed converter and its design method.

\section{Steady-state Analysis}

The LV stage of the CF-TIBCR consists of the main inductors $\left(L_{\mathrm{m} 1}\right.$ and $\left.L_{\mathrm{m} 2}\right)$, the switches $\left(S_{\mathrm{B} 1}\right.$ and $\left.S_{\mathrm{B} 2}\right)$, the anti-parallel diodes $\left(D_{\mathrm{SB} 1}\right.$ and $\left.D_{\mathrm{SB} 2}\right)$ and the resonant components $\left(L_{\mathrm{r}}, C_{\mathrm{SB} 1}\right.$ and $\left.C_{\mathrm{SB} 2}\right)$. The HV stage switches $\left(S_{\mathrm{F} 1} \sim S_{\mathrm{F} 4}\right)$ are combined with the resonant capacitances $\left(C_{\mathrm{SF} 1} \sim C_{\mathrm{SF} 4}\right)$ and the anti-parallel diodes $\left(D_{\mathrm{SF} 1} \sim D_{\mathrm{SF} 4}\right) . L_{\mathrm{H}}$ indicates the filter inductor to reduce the current ripple on the HV stage. TR is the high frequency transformer. A number of assumptions are needed to perform a simple analysis;

1) The main inductance, the filter inductance and the filter capacitance are significantly large. Therefore, the currents and voltages of the input and output are constant and ripple-free in one switching period.

2) The magnetizing inductance of TR is infinitely larger than $L_{\mathrm{r}}$. Thus, the magnetizing inductance does not affect the resonance.

3) The parasitic impedance, such as line impedance, switch conduction resistance, and transformer winding capacitance, are all neglected in the analysis.

Fig. 2 shows the key waveforms of the CF-TIBCR. The duty ratio of the LV stage switches gate signal $G_{\mathrm{SB}}$ is larger than $50 \%$ for the continuous flow of the main inductor currents. Also, the LV stage switches are controlled as shifting the phase of the gate signals which have a difference of 180 degrees. Thus, no interval exists in which both switches are turned off. According to the number of the resonant cycles, the proposed converter is operated as a one-cycle mode or a two-cycle mode as shown in Fig. 2(a) and (b). To satisfy the soft-switching condition, the LV

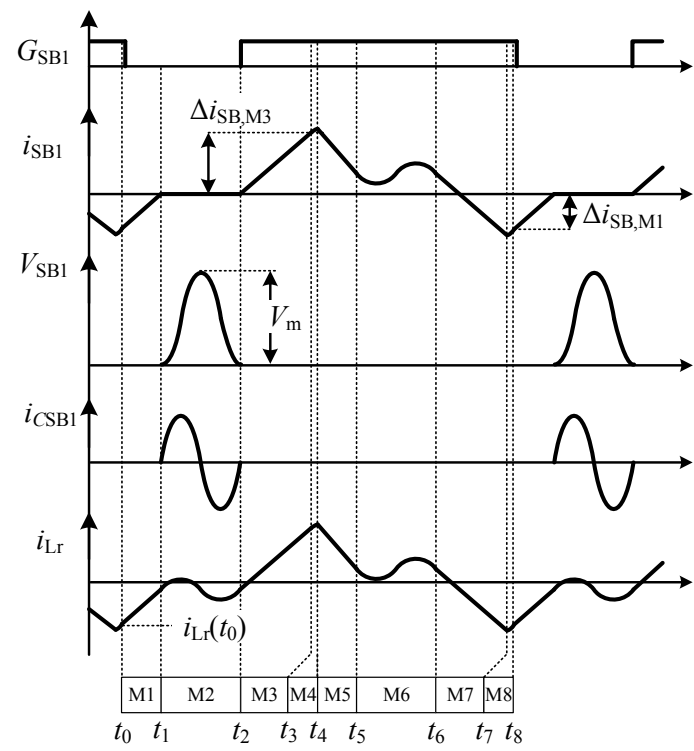

(a)

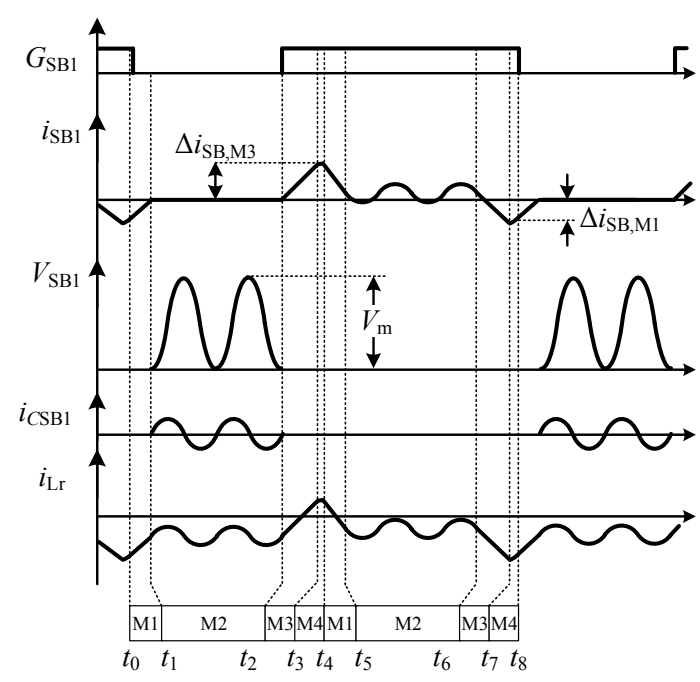

(b)

Fig. 2. Key waveforms of the CF-TIBCR in (a) the onecycle mode and (b) the two-cycle mode

stage switches should be turned-on at the end of the resonance in both operating modes. In case of the HV stage switches, they are turned-on when the LV stage switches are turned-off with dead time to satisfy the ZVS condition in the HV stage.

A switching cycle can be divided into eight intervals according to the resonant current. Since the same intervals symmetrically occur in each leg, only four intervals are analyzed (i.e., interval 1 to 4 ) in this paper.

Interval $1\left(t_{0} \leq t<t_{1}\right)$ : At $t_{0}$, the resonances between $L_{\mathrm{r}}$ and $C_{\mathrm{SF} 1} \sim C_{\mathrm{SF} 4}$ are finished. In addition, the current flowing through the switch $S_{\mathrm{B} 1}$ (i.e., $i_{\mathrm{SB} 1}$ ) starts to flow through the anti-parallel diode $D_{\mathrm{SB} 1}$ in this interval. Thus, the voltage of switch $S_{\mathrm{B} 1}$ during interval 1 is zero. The voltage of the transformer in the LV stage $V_{\mathrm{T} 1}$ is applied across the 
resonant inductor $L_{\mathrm{r}}$. Therefore, $i_{\mathrm{Lr}}$ starts to increase linearly, which can be described as:

$$
i_{\mathrm{Lr}}(t)=\frac{V_{\mathrm{H}}}{n_{\mathrm{T}} L_{\mathrm{r}}} t+i_{\mathrm{Lr}}\left(t_{0}\right)
$$

where $i_{\mathrm{Lr}}\left(t_{0}\right)$ is the initial resonant current at $t_{0}$ and $n_{\mathrm{T}}$ is the transformer turns ratio. When $i_{\mathrm{SB} 1}$ reaches zero, this interval ends. Using (1), the time interval $T_{\mathrm{M} 1}\left(t_{0}, t_{1}\right)$ can be expressed as:

$$
T_{\mathrm{M} 1}=\frac{L_{\mathrm{r}} \Delta i_{\mathrm{SB}, \mathrm{M} 1}}{V_{\mathrm{T} 1}}=\frac{n_{\mathrm{T}} L_{\mathrm{r}}\left|i_{\mathrm{Lr}}\left(t_{0}\right)+I_{\mathrm{Lm} 1}\right|}{V_{\mathrm{H}}}
$$

where $\Delta i_{\mathrm{SB}, \mathrm{M} 1}$ is an increment of $i_{\mathrm{SB} 1}(t)$, which is the difference between $i_{\mathrm{Lr}}\left(t_{0}\right)$ and $I_{\mathrm{Lm} 1}$.

Interval $2\left(t_{1} \leq t<t_{2}\right)$ : In this interval, the resonance occurs in the LV stage. At $t_{1}$, the current $i_{\mathrm{SB} 1}$ reaches zero, and starts to flow into the $C_{\mathrm{SB} 1}$. Since the $V_{\mathrm{T} 1}$ is applied across $L_{\mathrm{r}}$, the maximum voltage of $V_{\mathrm{SB} 1}\left(\right.$ i.e., $\left.V_{\mathrm{m}}\right)$ is twice that of $V_{\mathrm{T} 1}$. Using the above assumptions, $i_{\mathrm{Lr}}$ and voltage across the LV stage switch $V_{\mathrm{SB} 1}$ can be calculated as:

$$
\begin{aligned}
& i_{\mathrm{Lr}}(t)=\frac{V_{\mathrm{T} 1}}{Z_{\mathrm{NL}}} \sin \omega_{\mathrm{rL}}\left(t-t_{1}\right)-I_{\mathrm{Lm}} \\
& V_{\mathrm{SB} 1}(t)=V_{\mathrm{T} 1} \cos \omega_{\mathrm{rL}}\left(t-t_{1}\right)-V_{\mathrm{T} 1}
\end{aligned}
$$

where $\omega_{\mathrm{rL}}$ is the angular resonant frequency, and $Z_{\mathrm{NL}}$ is the characteristic impedance between $L_{\mathrm{r}}$ and $C_{\mathrm{SB} 1}$. The resonance ends when switch $S_{\mathrm{B} 1}$ is turned-on. To satisfy the ZVS condition, $S_{\mathrm{B} 1}$ should be turned-on when $V_{\mathrm{SB} 1}$ reaches zero. Therefore, the time interval $T_{\mathrm{M} 2}\left(t_{1}, t_{2}\right)$ is relative to the resonant period $T_{\mathrm{rL}}$ and the number of the resonant cycles $n_{\mathrm{r}}$.

$$
T_{\mathrm{M} 2} \approx T_{\mathrm{rL}} n_{\mathrm{r}}=2 \pi n_{\mathrm{r}} \sqrt{L_{\mathrm{r}} C_{\mathrm{SB} 1}}
$$

Interval $3\left(t_{2} \leq t<t_{3}\right)$ : Since switch $S_{\mathrm{B} 1}$ is turned-on at $t_{2}$, the current $i_{\mathrm{SB} 1}$, which is the difference between $i_{\mathrm{Lr}}$ and $I_{\mathrm{Lm}}$, flows through the switch $S_{\mathrm{B} 1}$. Similarly to the interval $1, i_{\mathrm{Lr}}$ is linearly increased because $V_{\mathrm{T} 1}$ is applied across $L_{\mathrm{r}}$. Therefore, $i_{\mathrm{Lr}}$ can be expressed as (6).

$$
i_{\mathrm{Lr}}(t)=\frac{V_{\mathrm{H}}}{n_{\mathrm{T}} L_{\mathrm{r}}}\left(t-t_{2}\right)-i_{\mathrm{Lr}}\left(t_{2}\right)
$$

This interval ends when the HV stage is turned-off. In the steady state, the difference between the increment of $i_{\mathrm{Lr}}$ in interval 1 and 3 is the input current $I_{\mathrm{L}}$. The time interval $T_{\mathrm{M} 3}\left(t_{2}, t_{3}\right)$ can be calculated as (7).

$$
T_{\mathrm{M} 3}=\frac{L_{\mathrm{r}} \Delta i_{\mathrm{SB}, \mathrm{M} 3}}{V_{\mathrm{T} 1}}=-\frac{L_{\mathrm{r}}\left\{i_{\mathrm{Lr}}\left(\mathrm{t}_{0}\right)-I_{\mathrm{Lm}}\right\}}{V_{\mathrm{T} 1}}
$$

Interval $4\left(t_{3} \leq t<t_{4}\right)$ : The resonances between $L_{\mathrm{r}}$ and the $\mathrm{HV}$ stage resonant capacitors $\left(C_{\mathrm{SF} 1} \sim C_{\mathrm{SF} 4}\right)$ occur in interval 4. In this interval, $i_{\mathrm{Lr}}$ is divided into two resonant routes. The voltage across the HV stage switch $V_{\mathrm{SF}}$ and $i_{\mathrm{Lr}}$ can be described as:

$$
\begin{aligned}
i_{\mathrm{Lr}}(t)= & i_{\mathrm{Lr}}\left(t_{3}\right) \cos \left\{\frac{\omega_{\mathrm{rH}}}{n_{\mathrm{T}}}\left(t-t_{3}\right)\right\}+\frac{V_{\mathrm{H}}}{Z_{\mathrm{NH}}} \sin \left\{\frac{\omega_{\mathrm{rH}}}{n_{\mathrm{T}}}\left(t-t_{3}\right)\right\} \\
V_{\mathrm{SF}}(t)= & \frac{V_{\mathrm{H}}}{2} \cos \left\{\frac{\omega_{\mathrm{rH}}}{n_{\mathrm{T}}}\left(t-t_{3}\right)\right\} \\
& -\frac{i_{\mathrm{Lr}}\left(t_{3}\right) Z_{\mathrm{NH}}}{2} \sin \left\{\frac{\omega_{\mathrm{rH}}}{n_{\mathrm{T}}}\left(t-t_{3}\right)\right\}+\frac{V_{\mathrm{H}}}{2}
\end{aligned}
$$

where $Z_{\mathrm{NH}}$ is the characteristic impedance and $\omega_{\mathrm{rH}}$ is the angular resonant frequency between $L_{\mathrm{r}}$ and $C_{\mathrm{SFx}}$ in the $\mathrm{HV}$ stage. Because calculating time of interval 4 using (9) is complicated, an approximated interval 4 (i.e., $T_{\mathrm{rH}, \mathrm{a}}$ ) can be calculated using (10). It can be derived by assuming that the resonant inductance $L_{\mathrm{r}}$ is significantly larger than the resonant capacitances in the HV stage.

$$
T_{\mathrm{rH}, \mathrm{a}}=\frac{2 n_{\mathrm{T}} C_{\mathrm{SF}} V_{\mathrm{H}}}{i_{\mathrm{Lr}}\left(t_{3}\right)}
$$

To achieve the ZVS condition in the HV stage, sufficient time is required to discharge the energy in the HV stage capacitors. Thus, the dead time of the HV stage switches $d_{\mathrm{D}}$ should be larger than the calculated value of $T_{\mathrm{rH}, \mathrm{a}}$.

When the CF-TIBCR operates in the buck operation, the gate signals remain the same. Thus, the operation modes including the equations of voltage and current are similar to a boost operation. All switches also have a soft-switching condition in the buck operation. Therefore, a mode analysis of the buck operation is omitted in this paper.

The voltage transfer ratio in the boost and buck operation $\left(G_{\mathrm{V}, \mathrm{BST}}\right.$ and $\left.G_{\mathrm{V}, \mathrm{BK}}\right)$ can be solved as (11) and (12) respectively, by equating input and output energy.

$$
\begin{aligned}
G_{\mathrm{V}, \mathrm{BST}} & =\frac{n_{\mathrm{T}} f_{\mathrm{r}}}{n_{\mathrm{r}} f_{\mathrm{sw}}} \\
G_{\mathrm{V}, \mathrm{BK}} & =\frac{n_{\mathrm{r}} f_{\mathrm{sw}}}{n_{\mathrm{T}} f_{\mathrm{r}}}
\end{aligned}
$$

As shown in (11) and (12), the output voltage of the proposed converter is determined by the number of resonant cycles and a ratio of the switching frequency to resonant frequency.

\section{Design of the CF-TIBCR}

The operating range of the CF-TIBCR is affected according to the resonant components because the duty 
ratio for satisfying soft-switching condition is restricted by the resonant components. Also, system efficiency mainly depends on the resonant components, since they mainly affect the peak current of the switches. Thus, we consider the available duty ratio and peak current according to the resonant components to achieve both soft-switching and increasing the operating range. In this paper, we use the basic system parameters where the rated power $P_{\text {rate }}$ is $300 \mathrm{~W}$, the low voltage is $24 V_{\mathrm{dc}}$, the range of the high voltage is $200 \sim 380 V_{\mathrm{dc}}$ and the transformer turns ratio is two.

\subsection{Duty Ratio to satisfy Soft-switching}

The turn-off time of the LV stage switches should include the resonant period and the interval of current flowing through the anti-parallel diode to achieve the ZVS conditions. Since the LV stage switches are controlled by overlapping, a duty of the LV stage switches $d_{\mathrm{SB}}$ should be within the range of 0.5 to 1 . In this Section, the lower limit of 0.6 and the upper limit of 0.9 are employed, considering the switch transition times. The duty ratio to satisfy a soft switching condition for the LV switches is calculated as (13), which is represented as $d_{\mathrm{SB}, \mathrm{BST}}$ or $d_{\mathrm{SB}, \mathrm{BK}}$ according to the operation mode.

$$
\begin{aligned}
& d_{\mathrm{SB}, \mathrm{BST}}=\frac{3}{4}-\frac{n_{\mathrm{r}} f_{\mathrm{sw}}}{2 f_{\mathrm{r}}}+\frac{n_{\mathrm{T}}^{2} f_{\mathrm{r}} L_{\mathrm{r}}\left|I_{\mathrm{H}}\right|}{2 n_{\mathrm{r}} V_{\mathrm{H}}}+d_{\mathrm{D}} \\
& d_{\mathrm{SB}, \mathrm{BK}}=\frac{3}{4}-\frac{n_{\mathrm{r}} f_{\mathrm{sw}}}{2 f_{\mathrm{r}}}-\frac{n_{\mathrm{T}}^{2} f_{\mathrm{r}} L_{\mathrm{r}}\left|I_{\mathrm{H}}\right|}{2 n_{\mathrm{r}} V_{\mathrm{H}}}+d_{\mathrm{D}}
\end{aligned}
$$

Figs. 3(a) and (b) illustrate $d_{\mathrm{SB}}$ with varying $L_{\mathrm{r}}$ and $C_{\mathrm{SBx}}$ in the boost operation. The peak current of the LV switches increases as the resonant inductance decreases or $V_{\mathrm{H}}$ increases. It leads to reduce duty ratio because $T_{\mathrm{M} 1}$, which is the time of current flowing through the anti-parallel diode, is increased. As shown in Fig. 3(a), $d_{\mathrm{SB}, \mathrm{BST}}$ can

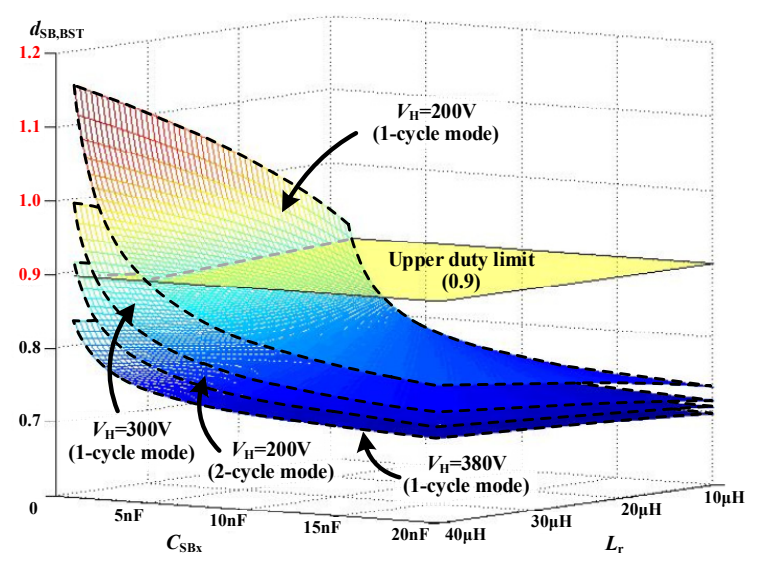

(a) exceed the upper duty limit when the output voltage is lower at the same load condition because $T_{\mathrm{M} 1}$ is reduced by decreasing of the slope of $i_{\mathrm{Lr}}$. Fig. 3(b) shows the required $d_{\mathrm{SB}, \mathrm{BST}}$ at an output voltage of $200 V_{\mathrm{dc}}$. In terms of the output power, the required $d_{\mathrm{SB}, \mathrm{BST}}$ of a high output power condition is higher than that of a low output power condition. Since the increment of input current makes the peak current of the switches higher, $T_{\mathrm{M} 3}$, which is the time of current flowing through the switches $S_{\mathrm{B}}$, becomes longer at the same inductance. As shown in Figs. 3(a) and (b), the duty ratio of the two-cycle mode is lower than that of the one-cycle mode because $T_{\mathrm{M} 2}$ (i.e., total resonant period) is increased although $T_{\mathrm{M} 1}$ is decreased. Therefore, when the converter is operated in the one-cycle mode in which the output voltage is low and the power condition is high, it is hard for $d_{\mathrm{SB}, \mathrm{BST}}$ to satisfy the duty consideration rather than the other conditions.

Figs. 4(a) and (b) depict $d_{\mathrm{SB}, \mathrm{BK}}$ curves with varying $L_{\mathrm{r}}$ and $C_{\mathrm{SBx}}$ in the buck operation. As $L_{\mathrm{r}}$ increases, $T_{\mathrm{M} 3}$ decreases because $T_{\mathrm{M} 1}$ and $T_{\mathrm{M} 3}$ are reversed in comparison to the boost operation. Thus, $d_{\mathrm{SB}, \mathrm{BK}}$ rapidly decreases in a low $V_{\mathrm{H}}$ condition due to the decrement of the slope $i_{\mathrm{Lr}}$ as shown in Fig. 4(a). In contrast to the boost operation, the higher output power makes $T_{\mathrm{M} 1}$ longer because of the increasing negative switch current. Thus, the turn off time, which is the sum of $T_{\mathrm{M} 1}$ and $T_{\mathrm{M} 2}$, is required more for a high output power condition as shown in Fig. 4(b). Although $T_{\mathrm{M} 2}$ is increased in the two-cycle mode, the duty ratio of the two-cycle mode is higher than that of the one-cycle mode because the $T_{\mathrm{M} 1}$ is significantly decreased. Therefore, the low input voltage and high power condition in the one-cycle mode can make $d_{\mathrm{SB}, \mathrm{BK}}$ exceed the lower duty limit similarly to the boost operation.

In both operation modes, the required duty is also affected by variation of $V_{\mathrm{L}}$. Since the required duty is determined by the voltage transfer ratio as (13), the lower $V_{\mathrm{L}}$ makes decrease the $f_{\text {sw }}$ in the same $V_{\mathrm{H}}$ condition, and

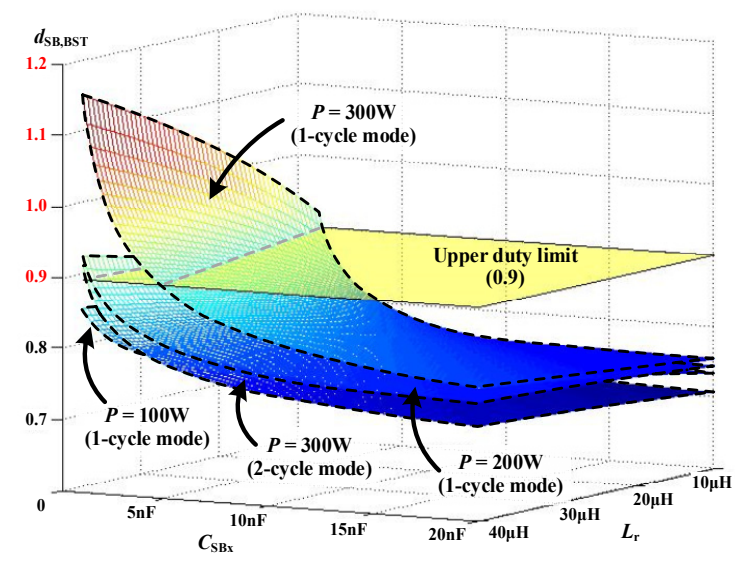

(b)

Fig. 3. Duty ratio of the LV stage switches to satisfy the soft switching condition according to (a) output voltage and (b) load condition in the boost operation 
it results increasing of the required duty. As a result, the resonant components should be designed, considering that $d_{\mathrm{SB}}$ does not exceed the duty limit considering the conversion ratio and load condition.

\subsection{Switch peak current}

When designing the proposed converter, it is important to consider the peak voltage and current in order to avoid device failure. High peak current can reduce system efficiency, because it increases the root mean square (RMS) value of the current. In the steady state, $i_{\mathrm{SB}, \mathrm{pk}}$ in the boost operation can be calculated as (14), determined by (6) and (13).

$$
i_{\mathrm{SB}, \mathrm{pk}}=\frac{V_{\mathrm{H}}}{4 n_{\mathrm{T}} L_{\mathrm{r}}}\left(\frac{1}{f_{\mathrm{sw}}}-\frac{2 n_{\mathrm{T}}}{f_{\text {res }}}\right)+\frac{P}{2 V_{\mathrm{L}}}
$$

In the buck operation, $i_{\mathrm{SB}, \mathrm{pk}}$ is a negative value with the same magnitude as that in the boost operation. Fig. 5

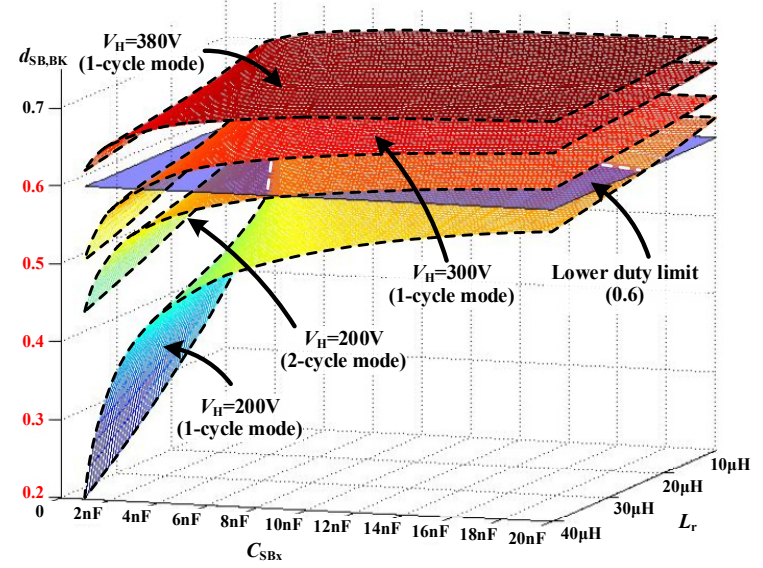

(a) shows $i_{\mathrm{SB}, \mathrm{pk}}$ waveforms of LV stage switches when $V_{\mathrm{H}}=380 \mathrm{~V}$ according to $L_{\mathrm{r}}$ and $C_{\mathrm{SBx}}$. Since the resonant inductance determines the slope of the $i_{\mathrm{SB}}$ for the whole switching period, $i_{\mathrm{SB}, \mathrm{pk}}$ is dramatically increased at a lower resonant inductance and higher $V_{\mathrm{H}}$ condition. As output power increases, the peak current increases due to longer $T_{\mathrm{M} 3}$. In both operations, the two-cycle mode has a larger peak value in comparison to the one-cycle mode.

\subsection{Load condition}

There is a maximum output power for the proposed converter to be able to satisfy the soft-switching condition (i.e., $P_{\mathrm{ss}, \max }$ ) depending on $f_{\mathrm{sw}}$ related with $V_{\mathrm{H}}$. If the output power of the converter is lower than $P_{\mathrm{ss}, \max }, T_{\mathrm{M} 1}$ exists because of the negative value of $i_{\mathrm{SB}}\left(t_{0}\right)$. However, $i_{\mathrm{SB}}\left(t_{0}\right)$ is larger than zero when the converter requires more power than $P_{\text {ss,max }}$. In this case, interval 1 does not exist throughout the whole switching period, and the resonant voltage does not reach zero. This leads to the failure of

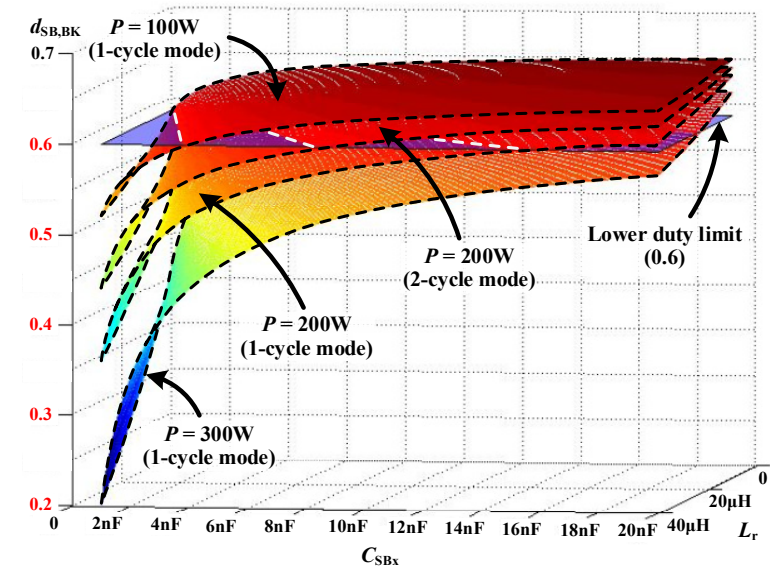

(b)

Fig. 4. Duty ratio of the LV stage switches to satisfy the soft switching condition according to (a) output voltage and (b) load condition in the buck operation

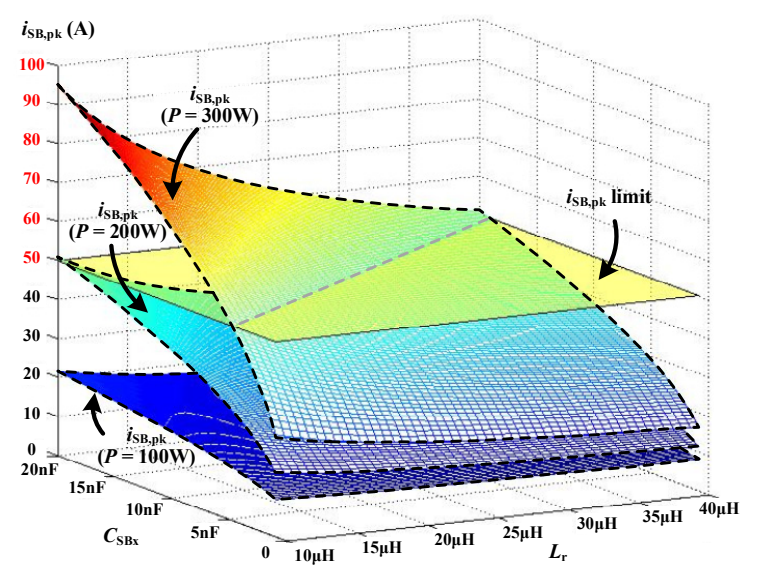

Fig. 5. Peak current of the LV stage switches according to the output power condition at $V_{\mathrm{H}}=380 V_{\mathrm{dc}}$ condition

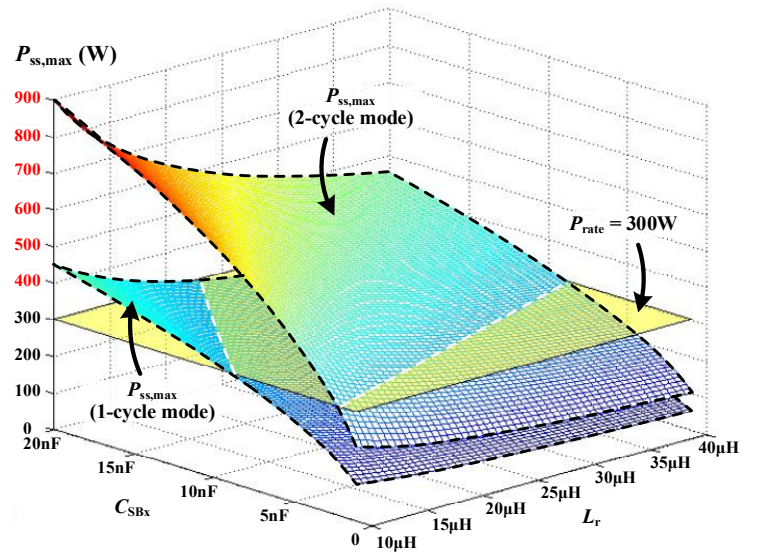

Fig. 6. Maximum output power to satisfy the soft-switching condition according to the resonant components 


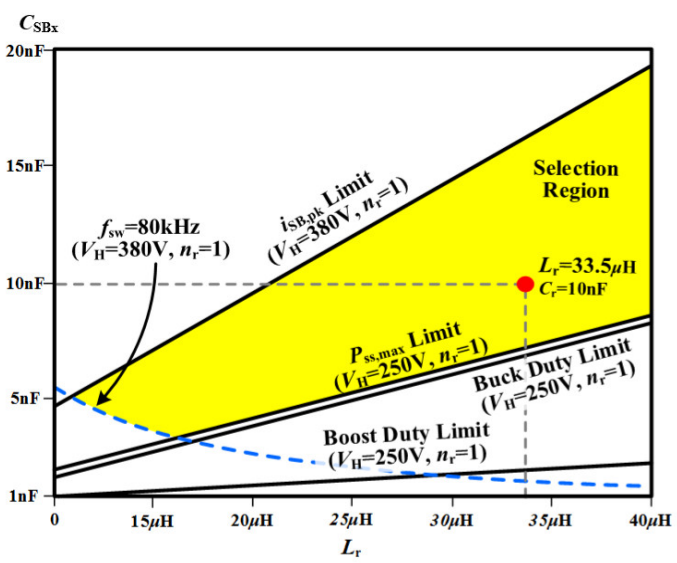

(a)

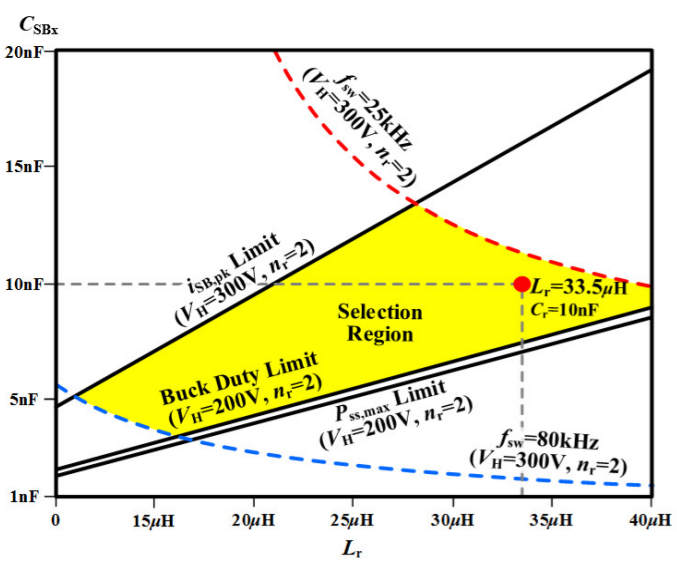

(b)

Fig. 7. Design examples of the resonant components considering $i_{\mathrm{SB}, \mathrm{pk}}, d_{\mathrm{SB}}$ and $P_{\mathrm{ss}, \max }$ conditions in (a) the one-cycle mode and (b) the two-cycle mode

the ZCS and ZVS. $P_{\text {ss, max }}$ can be calculated as (15) according to the resonant components, and it is depicted when voltage is low. This is the worst case for design and is shown in Fig. 6.

$$
P_{\mathrm{ss}, \max }=\frac{2 \pi n_{\mathrm{r}} V_{\mathrm{H}}^{2}}{n_{\mathrm{T}}^{2} Z_{\mathrm{NL}}}\left(0.5-\frac{n_{\mathrm{r}} f_{\mathrm{sw}}}{f_{\mathrm{res}}}-2 d_{\mathrm{D}}\right)
$$

If the resonant components are used in the region where the required output power is larger than $P_{\text {ss,max }}$, the converter cannot achieve a soft-switching conditions. Thus, $L_{\mathrm{r}}$ and $C_{\mathrm{SBx}}$ should be selected to fall within the region where the rated output power is lower than $P_{\text {ss,max }}$. As shown in Fig. 6, the higher characteristic impedance $Z_{\mathrm{NL}}$ and the one-cycle mode can cause a decrease in the $P_{\text {ss,max }}$. These factors make it difficult to satisfy the soft-switching conditions.

\subsection{Selection of the resonant components}

The converter operating the two-cycle mode is more

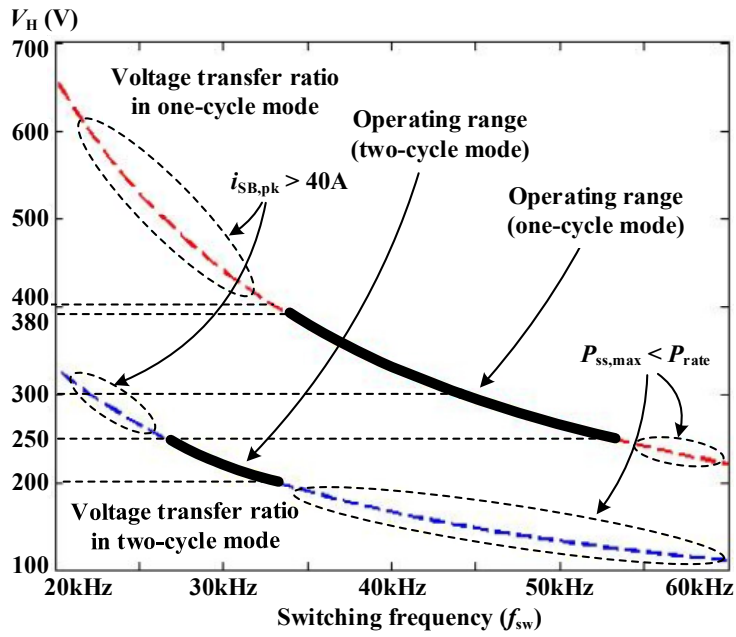

Fig. 8. Operating range of the proposed converter in each resonant cycle mode at full load condition

advantageous in the low voltage range because $d_{\mathrm{SB}}$ and $P_{\text {ss,max }}$ conditions, which satisfy the limits, widen although $i_{\mathrm{SB}, \mathrm{pk}}$ is higher. Thus, the resonant components are designed to satisfy both operation modes, which are the one-cycle mode in the high voltage range and the two-cycle mode in the low voltage range.

In this paper, the converter is operated as the one-cycle mode within the range from $250 V_{\mathrm{dc}}$ to $380 V_{\mathrm{dc}}$, and the twocycle mode under $250 V_{\mathrm{dc}}$. If the range of operating switching frequency is set between $25 \mathrm{kHz}$ and $80 \mathrm{kHz}$, the selection regions of the resonant components, which satisfy the above conditions, according to the operation mode are described as Fig. 7 using Figs. 3-6. There are four condition lines, representing the peak current limit, the $P_{\text {ss,max }}$ condition, and the duty limits of each mode. The softswitching condition is not satisfied when the resonant components are selected under the $P_{\text {ss,max }}$ condition line. Also, selection of the resonant components on the left side of the peak current limit line causes $i_{\mathrm{SB}, \mathrm{pk}}$ to become higher than $40 \mathrm{~A}$, which is the limit value. The trajectories of the resonant components related to switching frequency, which are the upper and lower frequency limits. Consequently, the resonant components should be designed to fall within the selection area bounded by the peak current limit, the $P_{\text {ss, max }}$ condition line, and the upper/lower switching frequency limits in each operation mode. In this paper, $L_{\mathrm{r}}=33.5 \mu \mathrm{H}$ and $C_{\mathrm{SB}}=10 \mathrm{nF}$ are chosen from the selection area to satisfy the limit conditions in both resonant cycle modes. In this case, the operation range of the converter is shown in Fig. 8. According to $V_{\mathrm{H}}$, the number of the resonance $n_{\mathrm{r}}$ is decided, and the required duty ratio $d_{\mathrm{SB}}$ is calculated to secure the time of the resonance depending on $n_{\mathrm{r}}$.

\section{Experimental Results}

To verify the proposed converter and its design method, 
a 300W laboratory prototype was built and tested. PQ4040 PL-7 and PQ3535 PL-7 core are used as the transformer and the resonant inductor, respectively. The transformer turns ratio is two. The proposed converter use TMS320F28335 (Texas Instrument) DSP-based digital controller, and FCH76N60NF (Fairchild Semiconductor) as the LV and HV stage switches. Also, all tests were measured using the LECROY WaveSurfer 24MXs. The system parameters are given in Table 1.

Figs. 9-12 show the key waveforms when the converter is operated in the boost operation with varying output

Table 1. Parameters of the prototype converter

\begin{tabular}{c|c|c|c}
\hline Parameter & Symbol & Value & Unit \\
\hline Low voltage & $V_{\mathrm{L}}$ & 24 & $\mathrm{~V}$ \\
\hline High voltage & $V_{\mathrm{H}}$ & $200 \sim 380$ & $\mathrm{~V}$ \\
\hline Power rating & $P_{\mathrm{rate}}$ & 300 & $\mathrm{~W}$ \\
\hline Switching frequency & $f_{\mathrm{sw}}$ & $20 \sim 80$ & $\mathrm{kHz}$ \\
\hline Main inductance & $L_{\mathrm{m}}$ & 1 & $\mathrm{mH}$ \\
\hline Resonant inductance & $L_{\mathrm{r}}$ & 33.5 & $\mu \mathrm{H}$ \\
\hline $\begin{array}{c}\text { Resonant capacitance } \\
\text { in the LV stage }\end{array}$ & $C_{\mathrm{SB}}$ & 10 & $\mathrm{nF}$ \\
\hline $\begin{array}{c}\text { Resonant capacitance } \\
\text { in the HV stage }\end{array}$ & $C_{\mathrm{SF}}$ & 1 & $\mathrm{nF}$ \\
\hline Transformer turns ratio & $n_{\mathrm{T}}$ & 2 & \\
\hline
\end{tabular}

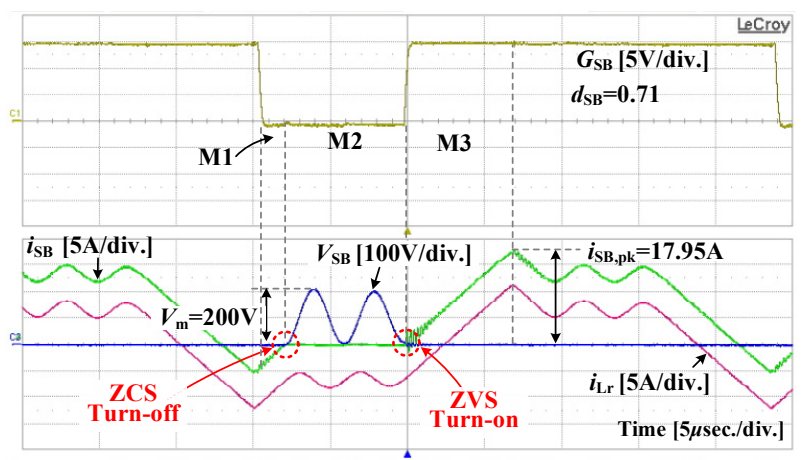

Fig. 9. The key waveforms of the CF-TIBCR at $200 V_{\mathrm{dc}}$ output voltage in the two-cycle mode of the boost operation

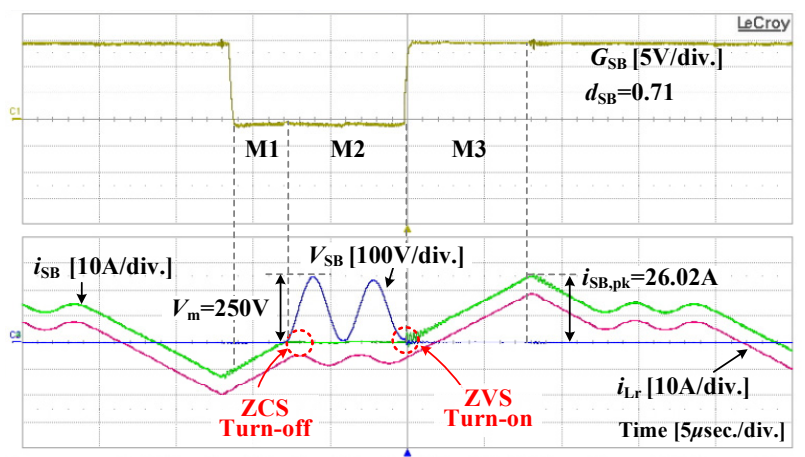

Fig. 10. The key waveforms of the CF-TIBCR at $250 V_{\mathrm{dc}}$ output voltage in the two-cycle mode of the boost operation voltages at full load condition. Since the turns ratio of the transformer is two, the peak voltage of the switches is the same as the output voltage. If the output voltage is lower than $250 V_{\mathrm{dc}}$, the converter using the designed resonant components cannot achieve the soft-switching condition in the one-cycle mode because the converter does not satisfy the $P_{\text {ss,max }}$ condition. Therefore, the converter should be operated in the two-cycle mode when the output voltage is lower than $250 V_{\mathrm{dc}}$. As shown in Figs. 9 and 10, the LV stage switches achieve ZVS turn-on and ZCS turn-off conditions in the two-cycle mode. The peak currents of the switches are measured at $17.95 \mathrm{~A}$ and $26.02 \mathrm{~A}$, whose calculated values by (14) are $18.05 \mathrm{~A}$ and $28.08 \mathrm{~A}$, respectively. These errors are caused by the parasitic components, such as the switch on-resistance, the forward voltage drop of the anti-parallel diode and the power loss of the magnetic components. Figs. 11 and 12 show that the converter is operated in the one-cycle mode that satisfies the soft-switching conditions. Using the one-cycle mode has the advantage of having a lower peak current value than the two-cycle mode in the same output voltage condition. For example, the switch peak current is measured at $15.20 \mathrm{~A}$ when the converter has an output voltage of $250 V_{\mathrm{dc}}$, which is almost $60 \%$ of the value of the converter operating in the two-cycle mode at the same

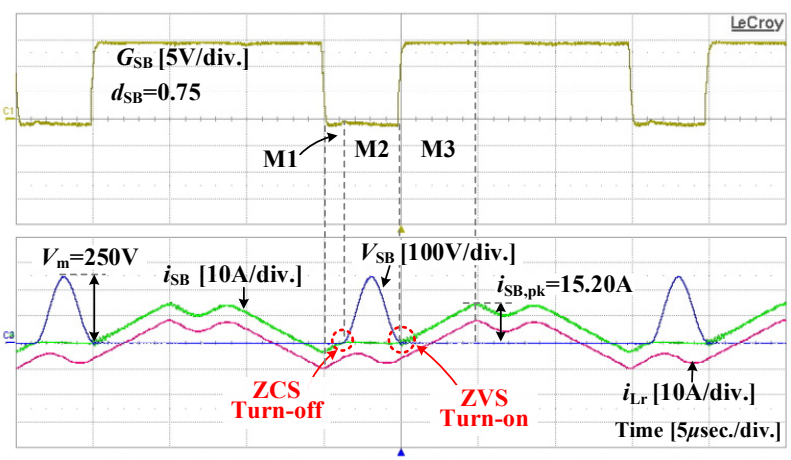

Fig. 11. The key waveforms of the CF-TIBCR at $250 V_{\mathrm{dc}}$ output voltage in the one-cycle mode of the boost operation

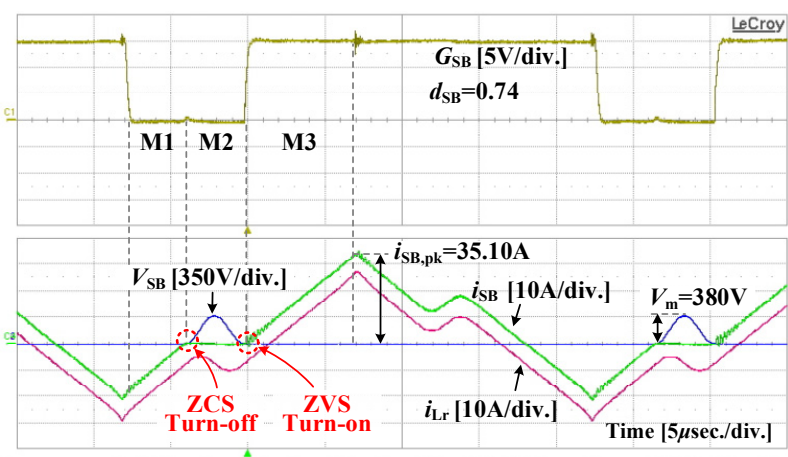

Fig. 12. The key waveforms of the CF-TIBCR at $380 V_{\mathrm{dc}}$ output voltage in the one-cycle mode of the boost operation 
output voltage.

Figs. 13-16 are the key waveforms of the converter, which is operated in the buck operation that varies the input voltage and has a fixed output voltage of $24 V_{\mathrm{dc}}$. In contrast with the boost operation, $i_{\mathrm{SB}}$ is smaller than $i_{\mathrm{Lr}}$ by $I_{\mathrm{Lm}}$, and $T_{\mathrm{M} 1}$ becomes longer than $T_{\mathrm{M} 3}$ in the buck operation because of the negative value of $I_{\mathrm{Lm}}$. Therefore, the maximum magnitude of the switch current occurs at $t_{0}$. In the low input voltage range, the converter is operated in the two-cycle mode to satisfy a duty limit condition as shown in Figs. 13 and 14. Similarly to the boost operation, the converter operating in the one-cycle mode has the advantage of the lower peak current when the input voltage is higher than $250 V_{\mathrm{dc}}$ as shown in Figs. 14 and 15. However, a magnitude of the negative peak current in the buck operation increases in the higher output voltage condition because of increment of $T_{\mathrm{M} 1}$. Therefore, the maximum negative current of the switches is measured by $-38.7 \mathrm{~A}$ at condition of the highest input voltage although the converter operates in the one-cycle mode as shown in Fig. 16. Also, a lower switching frequency is needed in the same resonant cycle mode along with increases in the input voltage.

In both operation modes, there are errors of duty ratio

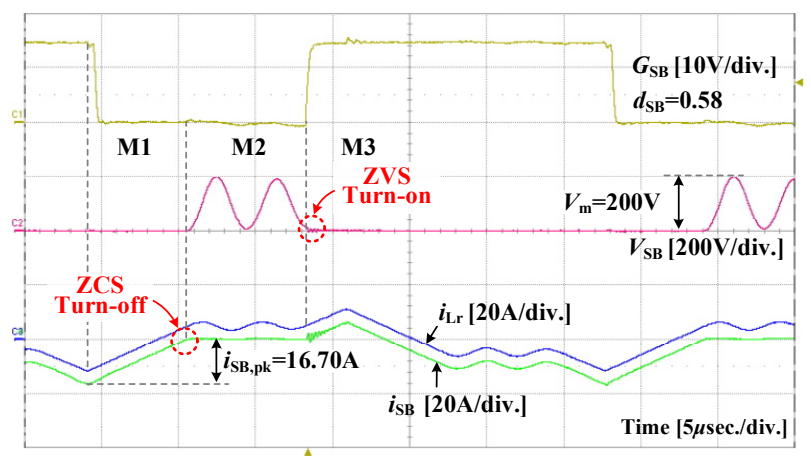

Fig. 13. The key waveforms of the CF-TIBCR at $200 V_{\mathrm{dc}}$ input voltage in the two-cycle mode of the buck operation

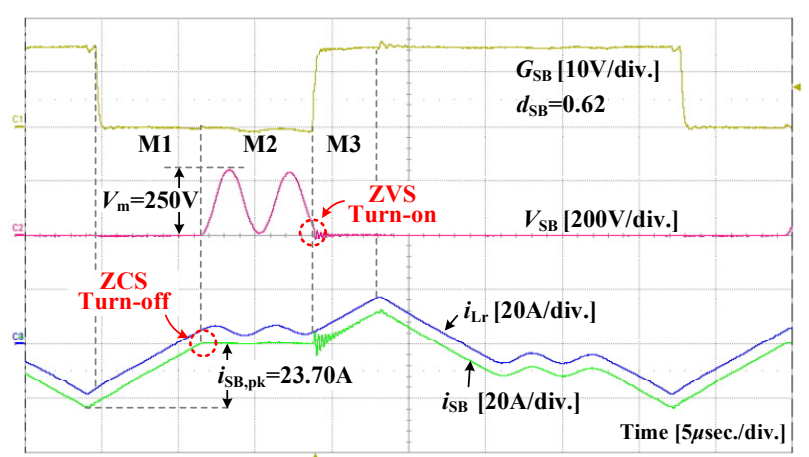

Fig. 14. The key waveforms of the CF-TIBCR at $250 V_{\mathrm{dc}}$ input voltage in the two-cycle mode of the buck operation between the calculated value using (13) and the used value in the experiment. The main reason is parasitic impedance on the current path, which decreases the slope and peak value of the switch current. It cannot enable the switch voltage to be equal to zero at the end of the resonance.

When the resonant mode is transferred, the operation switching frequency is varied by change of $n_{\mathrm{r}}$ according to (11) or (12). Also $d_{\mathrm{SB}}$ is changed depending on the output voltage. For example, if the output voltage reference exceeds $250 V_{\mathrm{dc}}$ in the boost operation, the operating frequency is decreased to almost half value of the switching frequency in one-cycle mode by the voltage controller. The duty ratio of the LV stage switches is also changed according to the switching frequency. Fig. 17 shows the experimental results when the resonant mode is changed. During the transient time, the output voltage is not exactly same as its reference value. In this case, the LV stage switches achieve the ZCS condition but it cannot satisfy the ZVS condition, because the duty ratio is not enough to secure the time for the resonance. Therefore, there are some distortions in the voltage of the switch and current flowing through the resonant inductor as shown in Fig. 18.

Fig. 19 shows the efficiency curves of the proposed CF-

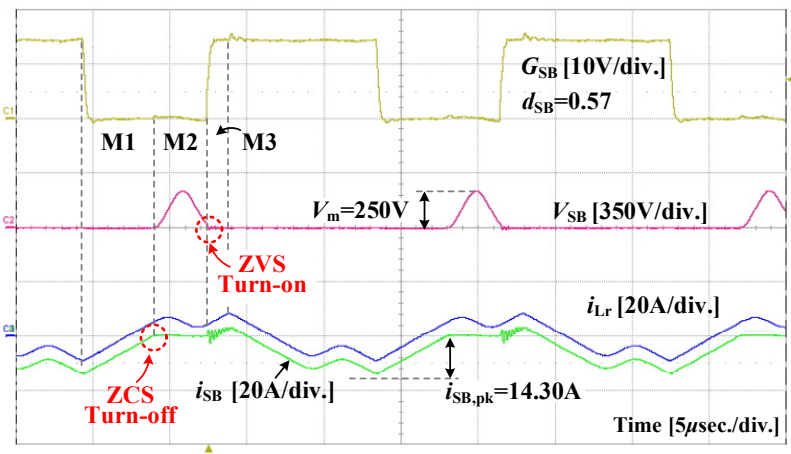

Fig. 15. The key waveforms of the CF-TIBCR at $250 V_{\mathrm{dc}}$ input voltage in the one-cycle mode of the buck operation

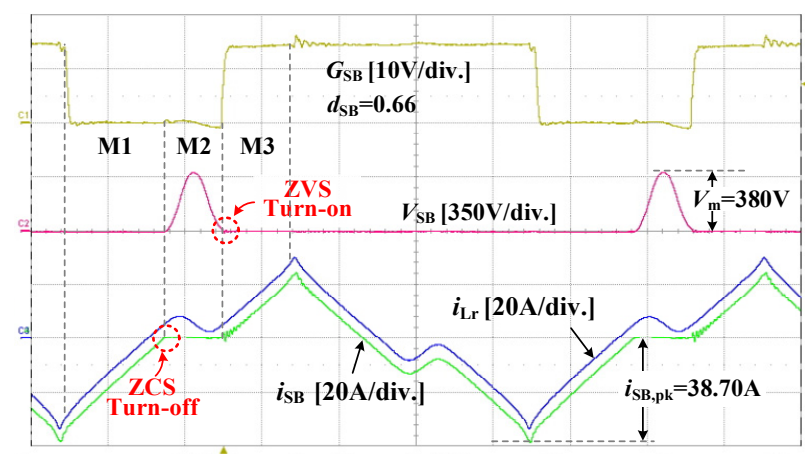

Fig. 16. The key waveforms of the CF-TIBCR at $380 V_{\mathrm{dc}}$ input voltage in the one-cycle mode of the buck operation 


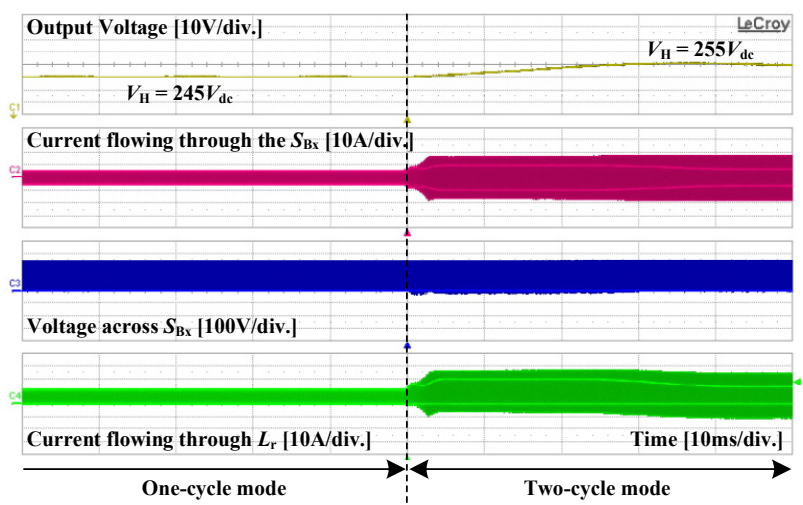

Fig. 17. The waveforms of the resonant mode transfer

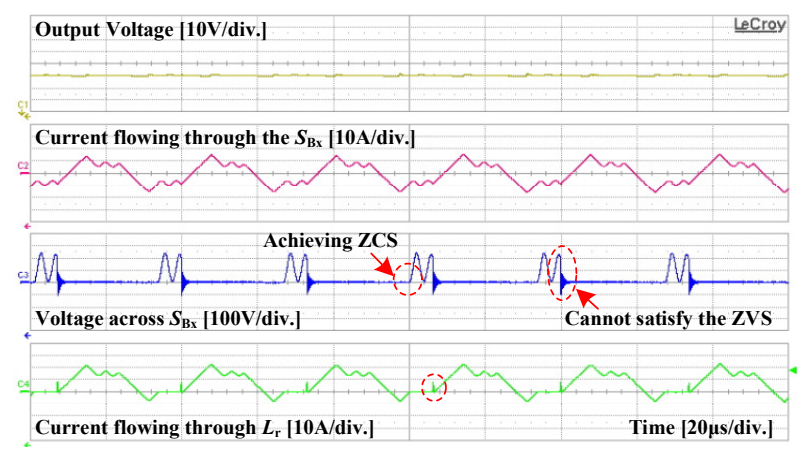

Fig. 18. The enlarged waveforms during the transient time

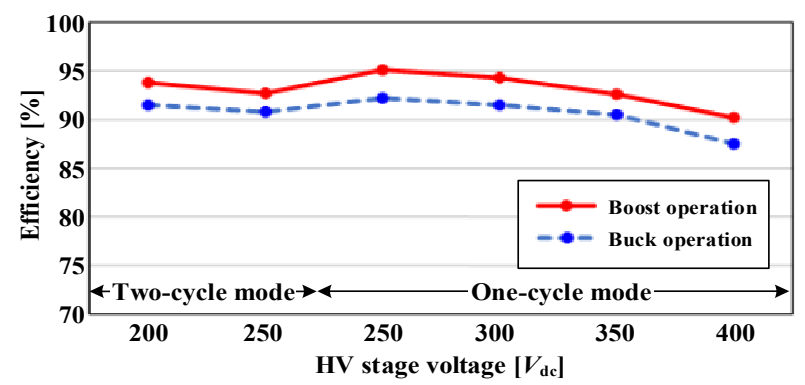

Fig. 19. The efficiency of the proposed converter

TIBCR according to $V_{\mathrm{H}}$. The maximum efficiency is measured at $95.1 \%$ in the boost operation. In the buck operation, it is also measured at $92.2 \%$. In the same resonant mode, the efficiency decreases with increasing $V_{\mathrm{H}}$. This is because the slope of $i_{\mathrm{SB}}$ during $T_{\mathrm{M} 1}$ and $T_{\mathrm{M} 3}$, which causes higher RMS value of switch current, becomes steep as $V_{\mathrm{H}}$ increases. In terms of the resonant mode, the efficiency in the one-cycle is higher than that of the twocycle mode, because of the smaller $i_{\mathrm{SB}, \mathrm{pk}}$ and the higher switching frequency in the one-cycle mode. In the buck operation, the efficiency is commonly lower than that of the boost operation because of the longer $T_{\mathrm{M} 1}$. That is why the errors of $i_{\mathrm{SB}, \mathrm{pk}}$ between the calculated value and the experimental result are higher than that of the boost operation.

\section{Conclusion}

This paper proposes a current-fed bi-directional DC/DC converter using resonance and its design method to extend the voltage range. The proposed converter has a high input current rating and low current ripple because the LV stage current is divided into two inductors. In addition, the converter provides a high voltage transfer ratio with a low transformer turns ratio. Using the resonant components, the proposed converter solves the voltage spike problem, and achieves ZVS and ZCS conditions for the LV stage switches and ZVS condition for the HV stage switches. Soft-switching that varies the voltage is achieved through the control of the switching frequency and duty ratio. To extend the voltage range, the method of changing the number of the resonance is adopted. Also, the design method for the proposed converter is derived by considering the duty, peak current and $P_{\mathrm{ss}, \max }$ conditions. The experiments using the $300 \mathrm{~W}$ laboratory prototype in both the operation modes reveal good performance, which verifies the proposed method.

\section{Acknowledgements}

This work was supported by the Human Resources Program in Energy Technology of the Korea Institute of Energy Technology Evaluation and Planning (KETEP) granted financial resource from the Ministry of Trade, Industry \& Energy, Republic of Korea (No. 2016 4030200980)

This research was supported by Basic Science Research Program through the National Research Foundation of Korea (NRF) funded by the Ministry of Science, ICT \& Future Planning (No. 2014R1A2A2A05006744).

\section{References}

[1] Fontes, G., Turpin, C., Astier, S. and Meynard, T.A., "Interactions Between Fuel Cells and Power Converters: Influence of Current Harmonics on a Fuel Cell Stack," IEEE Trans. Power Electronics, vol. 22, no. 2, pp. 670-678, Mar. 2007.

[2] Blaabjerg, F., Zhe Chen and Kjaer, S.B., "Power electronics as efficient interface in dispersed power generation systems," IEEE Trans. Power Electronics, vol. 19, no. 5, pp. 1184-1194, Sept. 2004.

[3] Araujo, S.V., Torrico-Bascope, R.P. and TorricoBascope, G.V., "Highly Efficient High Step-Up Converter for Fuel-Cell Power Processing Based on Three-State Commutation Cell," IEEE Trans. Industrial Electronics, vol. 57, no. 6, pp. 1987-1997, Jun. 2010.

[4] Wolfs, P. J., Ledwich, G. F., and Kwong, K. C., "The application of the duality principle to nonplanar circuits," IEEE Trans. Power Electronics, vol. 8, no. 
2, pp. 104-111, Apr. 1993.

[5] Wolfs, P. J., "A current-sourced DC-DC converter derived via the duality principle from the half-bridge converter," IEEE Trans. Industrial Electronics, vol. 40, no. 1, pp. 139-144, Feb. 1993.

[6] Sang-Kyoo Han, Hyun-Ki Yoon, Gun-Woo Moon, and Myung-Joong Youn, Yoon-Ho Kim and KangHee Lee, "A new active clamping zero-voltage switching PWM current-fed half-bridge converter," IEEE Trans. Power Electronics, vol. 20, no. 6, pp. 1271-1279, Nov. 2005.

[7] S. J. Jang, , Chung-Yuen Won, Byoung-kuk Lee and Jin Hur, "Fuel Cell Generation System With a New Active Clamping Current-Fed Half-Bridge Converter," IEEE Trans. Energy Conversion, vol. 22, no. 2, pp. 332-340, Jun. 2007.

[8] Quan Li; Wolfs, P., “A Current Fed Two-Inductor Boost Converter With an Integrated Magnetic Structure and Passive Lossless Snubbers for Photovoltaic Module Integrated Converter Applications," IEEE Trans. Power Electronics, vol. 22, no. 1, pp. 309-321, Jan. 2007.

[9] Ren-Yi Chen, Tsorng-Juu Liang, Jiann-Fuh Chen, Ray-Lee Lin and Kuo-Ching Tseng, "Study and Implementation of a Current-Fed Full-Bridge Boost DC-DC Converter With Zero-Current Switching for High-Voltage Applications," IEEE Trans. Industry Applications, vol. 44, no. 4, pp. 1218-1226, July-Aug. 2008.

[10] Bo Yuan, Xu Yang, Xiangjun Zeng, J. Duan, J. Zhai and Donghao Li, "Analysis and design of a high stepup current-fed multiresonant DC-DC converter with low circulating energy and zero-current switching for all active switches," IEEE Trans. Industrial Electronics, vol. 59, no. 2, pp. 964-978, Feb. 2012.

[11] Bo Yuan, Xu Yang, Donghao Li, Yunqing Pei, Duan, J., Zhai, J., "A Current-Fed Multiresonant Converter with Low Circulating Energy and Zero-Current Switching for High Step-Up Power Conversion," IEEE Trans. Power Electronics, vol. 26, no. 6, pp. 16131619, Jun. 2011.

[12] Prasanna, P.R.; Rathore, A.K., "Analysis, Design, and Experimental Results of a Novel Soft-Switching Snubberless Current-Fed Half-Bridge Front-End Converter-Based PV Inverter," IEEE Trans. Power Electronics, vol. 28, no. 7, pp. 3219-3230, July 2013.

[13] Pan Xuewei, A. K. Rathore and U. R. Prasanna, "Novel soft-switching snubberless naturally clamped current-fed full-bridge front-end-converter-based bidirectional inverter for renewables, microgrid, and UPS applications," IEEE Trans. Industry Applications, vol. 50, no. 6, pp. 4132-4141, Nov.-Dec. 2014.

[14] Pan Xuewei and A. K. Rathore, "Novel bidirectional snubberless naturally commutated soft-switching current-fed full-bridge isolated DC/DC converter for fuel cell vehicles," IEEE Trans. Industrial Electronics, vol. 61, no. 5, pp. 2307-2315, May. 2014.
[15] Pan Xuewei and A.K. Rathore, "Novel interleaved bidirectional snubberless soft-switching current-fed full-bridge voltage doubler for fuel-cell vehicles," IEEE Trans. Power Electronics, vol. 28, no. 12, pp. 5535-5546, Dec. 2013.

[16] A.K. Rathore and U.R. Prasanna, "Analysis, design, and experimental results of novel snubberless bidirectional naturally clamped ZCS / ZVS current-fed half-bridge DC/DC converter for fuel cell vehicles," IEEE Trans. Industrial Electronics, vol. 60, no. 10, pp. 4482-4491, Oct. 2013.

[17] S.W Lee, S.R Lee and C.H Jeon, "A New High Efficient Bi-directional DC/DC Converter in the Dual Voltage System," Journal of Electrical Engineering \& Technology, vol. 1, no. 3, pp. 343-350, Sep. 2006.

[18] Hua Bai and Mi, C., "Eliminate Reactive Power and Increase System Efficiency of Isolated Bidirectional Dual-Active-Bridge DC-DC Converters Using Novel Dual-Phase-Shift Control," IEEE Trans. Power Electronics, vol. 23, no. 6, pp. 2905-2914, Nov. 2008.

[19] Biao Zhao, Qiang Song and Wenhua Liu, "Power Characterization of Isolated Bidirectional DualActive-Bridge DC-DC Converter With Dual-PhaseShift Control," IEEE Trans. Power Electronics, vol. 27, no. 9, pp. 4172-4176, Sept. 2012.

[20] Jiarong Kan, Shaojun Xie, Yu Tang and Yunya Wu, a "Voltage-Fed Dual Active Bridge Bidirectional DC/ DC Converter With an Immittance Network," IEEE Trans. Power Electronics, vol. 29, no. 7, pp. 35823590, July 2014.

[21] Radika. P, "A High Efficiency DC-DC Boost Converter with Passive Regenerative Snubber," Journal of Electrical Engineering \& Technology, vol. 9, no. 2, pp. 501-507, Mar. 2014.

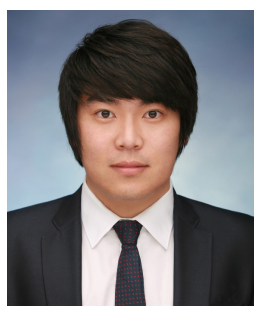

Yong-Su Noh He was born in Suwon, Korea in 1985. He received B.S. degree in electrical and electronic engineering from Ajou University, Suwon, Korea in 2011. He is currently working toward his Ph.D. degree in Electrical and Computer Engineering at Sungkyunkwan University, Suwon, Korea. His current research interests include DC/DC converters, resonant converter, and photovoltaic power generation.

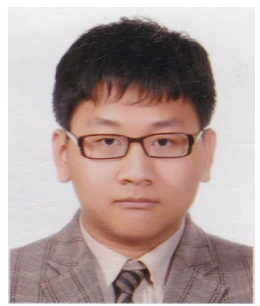

Bum-Jun Kim He was born in Seoul, Korea in 1992. He received B.S. degree in electrical and electronic engineering from Sungkyunkwan University, Suwon, Korea in 2015. He is currently working toward his M.S. degree in Electrical and Computer Engineering at Sung- 
kyunkwan University, Suwon, Korea. His current research interests include DC/DC converters, battery charger, and its digital control.

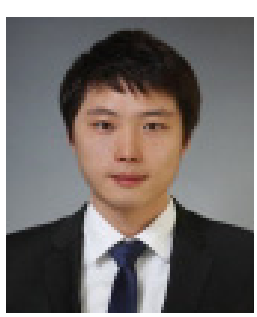

Seong-Chon Choi He received B.S. degree in electrical and electronic engineering from Kangwon University, Chuncheon, Korea in 2011, and the M.S degree in Electrical and Computer Engineering at Sungkyunkwan Univer sity, Suwon, Korea in 2013. He is currently working toward his Ph.D. degree in Electrical and Computer Engineering at Sungkyunkwan University, Suwon, Korea. His current research interests include DC/DC converters and battery management system and its robust control.

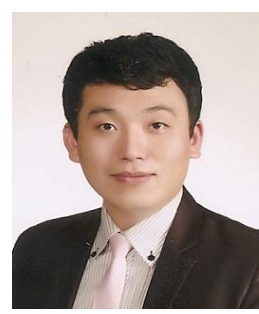

Do-Yun Kim He received B.S. degree in electrical and electronic engineering from Anyang University, Anyang, Korea in 2011, and the M.S degree in Interdisciplinary Program in Energy System Engineering at Sungkyunkwan University, Suwon, Korea in 2013. He is currently working toward his Ph.D. degree in Interdisciplinary Program in Photovoltaic System Engineering at Sungkyunkwan University, Suwon, Korea. His current research interests include inverter and battery management system and its robust control.

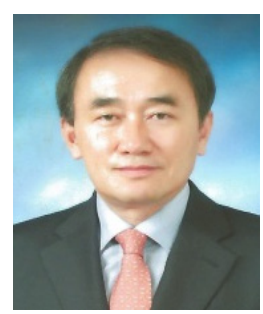

Chung-Yuen Won $\mathrm{He}$ was born in Korea in 1955. He received the B.S. degrees in Electrical Engineering from Sungkyunkwan University, Suwon, Korea, in 1978, and the M.S. and Ph.D. degrees in Electrical Engineering from Seoul National University, Seoul, Korea, in 1980 and 1987, respectively. From 1990 to 1991, he was with the Department of Electrical Engineering, University of Tennessee, Knoxville, as a Visiting Professor. Since 1988, he has been with a member of the faculty of Sungkyunkwan University, where he is a Professor in the College of Information and Communication Engineering; also he is the director of Samsung Energy Power Research Center. He was the President of the Korean Institute of Power Electronics in 2010. Since 2011, he has been a director of the Korean Federation of Science and Technology Societies. He is also head officer in Human Education Program for DC Distribution Electro technology from 2016. His current research interests include the power electronic of electric machines, electric / hybrid vehicle drives, power converters for renewable energy systems. He is a senior member of the Institute of Electrical and Electronics Engineers (IEEE). 